\title{
Congreso Internacional ARQUEOLOGÍA POSTMEDIEVAL EN ITALIA. ANTECEDENTES Y PERSPECTIVAS
}

(Sassari, 17-20 Octubre 1994)

\begin{abstract}
La progresiva ampliación del espacio temporal objeto de estudio de la arqueología en Europa, está ligado a una serie de motivos relacionados con el desarrollo de esta disciplina en los últimos decenios. Indudablemente, en el caso italiano, el desarrollo de la arqueología postclásica y de la arqueología de intervención han contribuido considerablemente a este avance. Igualmente la extensión de la arqueología estratigráfica ha tenido un importante papel en la valoración del depósito arqueológico de forma global. El ejemplo de Génova es significativo; de la mano del Instituto de Historia de la Cultura Material (I.S.C.U.M.) se ha realizado desde los años 60 una monumental actividad de arqueología urbana, tanto de urgencia como preventiva, que ha contribuido a la teorización de la archeología globale (Mannoni et alii 1988).
\end{abstract}

De todas formas, a pesar del fuerte crecimiento de las intervenciones arqueológicas postmedievales publicadas en Italia (73 en los años setenta, 189 en los ochenta y, como proyección, un número similar para el decenio actual), se observan una serie de profundos desequilibrios. Estos se manifiestan no solamente en el orden geográfico (tres cuartos de estas intervenciones se concentran en el norte del país), sino también en las áreas estudiadas: más del $70 \%$ pertenecen a intervenciones urbanas. Pero quizás el hecho más significativo sea que la gran mayoría de las intervenciones postmedievales italianas (la observación es también aplicable a la realidad hispana) tienen lugar como apéndice de actuaciones arqueológicas centradas en el periodo medieval o anterior, de manera que no responden a problemáticas de investigación propias de este periodo. El peligro es pues, que la arqueología postmedieval se desarrolle como una mera extensión de la arqueología medieval, sin reflexionar sobre los contenidos, las problemáticas e incluso las metodologías a emplear en un contexto diferente. Como resultado de esta tendencia, que contrasta profundamente con la madurez que progresivamente ha adquirido la arqueología medieval, parece lícito preguntarse si verdaderamente existe en Italia una arqueología de las sociedades modernas y contemporáneas, y en el caso de respuesta afirmativa, como viene realizada, con que finalidades, y con cuales estrategias y metodologías de estudio.

La situación del panorama europeo es muy desigual, pero existen experiencias maduras y puntos de referencia a tener en cuenta. Sobre todo es en los paises nordoccidentales donde la arqueología postmedieval se ha desarrollado de forma mas decidida. De hecho, y de la misma forma que había sucedido ya con la arqueología medieval, ha sido Gran Bretaña el país pionero en el desarrollo de la arqueología postmedieval, y la aparición de la revista anual Postmedieval Archaeology' en el año 1967 es un buen indicador de su madurez. En el caso francés la revista RAMAGE (Revue d'Archéologie Moderne et Generale) ${ }^{2}$ viene apareciendo desde 1981.

I.- Revista publicada por la Society for Post-Medieval Archaeology, Departament of Medieval and Later Antiquites, The British Museum, Great Russell Street, GB London WC IB3DC.

2.- Revista publicada por el Centre D'Archeologie du Monde Moderne, Universite Paris Sorbonne, 3 rue Michelet, F. 75006 París. 
Por último, la revista de arqueología postmedieval checoslovaca ha aparecido en 1990 bilingüe en inglés.

Con la intención de promover una reflexión madura y global sobre los problemas planteados por la práctica de esta nueva disciplina, se han organizado en Italia durante el año 1994 dos congresos de forma paralela, uno sobre cultura material, y otro sobre el desarrollo disciplinar y sus perspectivas de estudio.

El primero de ellos, bajo el título La cerámica postmedieval en Italia. La aportación de la arqueología, se celebró en Albisola Superiore (provincia de Savona) entre los dias 27 y 29 de mayo de 1994. Se trata del vigésimo séptimo congreso internacional de la cerámica organizado por el Centro Ligure per la Storia della Cerámica, en esta ocasión en colaboración con el Departamento de Historia de la Universidad de Sássari(3). Este encuentro, se ha propuso contribuir a la construcción de instrumentos de investigación arqueológica, sistematizando y sintetizando todos los conocimientos existentes sobre las producciones cerámicas postmedievales en las diversas regiones italianas. El encuentro se articuló en tres secciones (I. Arqueología y cerámica postmedieval, 2. Las situaciones regionales, 3. Indicadores cronológicos plurirregionales), con un total de 46 comunicaciones.

Después de la sección introductoria, que se dedicó al examen crítico del problema de la tutela de los contextos arqueológicos, el núcleo central del encuentro analizó las situaciones regionales, mostrando la desigualdad del desarrollo de la arqueología postmedieval en el territorio italiano. De todas formas, y a pesar de la heterogeneidad de fondo, un hilo conductor común guió todas las ponencias regionales, al analizar los problemas siguientes: I. Los centros productivos, 2. Las tipologías regionales, 3. Documentos arqueológicos que ilustrasen el consumo, 4. Las exportaciones.

Tuvieron especial incidencia las ponencias de la tercera sección, dedicadas a las producciones cerámicas que, por su notable expansión comercial, se han difundido por gran parte de la península italiana, configurándose como verdaderos indicadores cronológicos de gran interés. Incluso algunas producciones, como la maiolica ligur (V.AA. 1992) o la Taches Noires albisolese (Cameirana 1977), han alcanzado una distribución mercantil de ámbito internacional, interesando amplias zonas de la Península lbérica.

Naturalmente, hacer el balance de la situación actual de los estudios sobre la cultura postmedieval, aunque en este caso reducida a la cerámica, y sobre aspectos relativos a la estructura de la investigación, no era sino un pri- mer paso. Era necesario un intento de reflexión general con el fin de individualizar y formular directrices de investigación, reuniendo los datos de excavaciones y estudios aislados y generalmente fragmentarios, con el fin de dar vida a un debate científico que definiese las bases de la disciplina. Los límites cronológicos y las áreas de estudio, las relaciones con las otras ciencias humanas y naturales operantes en estos sectores cronológicos, la relación metodológica y temática con las "otras arqueologías", son algunos, entre otros aspectos, de los elementos a discutir y a matizar si queremos seguir hablando de arqueología postmedieval.

La existencia de realidades europeas que ya han abordado de diversa forma, y por tanto enriquecedora, estas problemáticas y discusiones, ha provocado la necesidad de enfocar un diálogo científico que tome como punto de partida las experiencias realizadas fuera de Italia.

A partir de estas premisas se ha organizado un nuevo congreso internacional en Sassari (Cerdeña) durante los dias 17 al 20 de Octubre de 1994. Bajo el título La arqueología postmedieval: La experiencia europea e Italia (4), el encuentro se propuso discutir la situación europea de esta disciplina, confrontándola con la realidad italiana. De hecho, el encuentro se ha propuesto valorar de forma paralela, tanto los casos europeos, como la vía italiana (en tantos aspectos similar a la española) hacia la arqueología postmedieval.

El congreso ha estado dividido en cuatro secciones, que han analizado de forma jerarquizada, desde las perspectivas más amplias a las más concretas, los aspectos constitutivos de la disciplina desde la visión italiana, para seguir con las síntesis europeas, con el fin de ofrecer una serie de claves de comprensión y definición de la arqueología postmedieval lo mas completas posible.

I. La definición de la disciplina, donde Tiziano Mannoni ha analizado el problema de la arqueología postmedieval en el contexto científico italiano, centrándose sobre todo en las relaciones existentes entre esta diciplina y la arqueología global. El desarrollo de esta visión abierta y diacrónica de la arqueología está en la base del nacimiento de algunas importantes experiencias italianas de arqueología urbana, medieval, y postmedieval, que explican gran parte de las particularidades de la arqueología postmedieval en este país.

2. El marco europeo, sección en la que diversos investigadores ofrecieron un panorama del desarrollo de la disciplina en el ámbito de Europa Occidental, que ofrece paralelos y afinidades de primer orden con la situación de la arqueología italiana; por ello no se han tenido en cuenta experiencias igualmente interesantes, como las de la arqueología histórica norteamericana.

3.- Las actas se encuentran actualmente en prensa y estarán disponibles antes de finales del presente año. Para mayor información conectar con el Centro Ligure per la Storia della Ceramica, presso Museo Storico-Archeologico Fortezza del Priamar, Corso Mazzini I, 17100 Savona (Italia).

4.- Ver nota 3 
La primera ponencia, la de D. Crossley (Current priorities in Post-Medieval archaeology in Britain) ha mostrado el resultado de mas de 30 años de trabajo en Gran Bretaña, que se traducen no sólo en la elaboración de afinados instrumentos de análisis arqueológico (tipologías constructivas, indicadores cronológicos, etc.), sino tambien en la definición de complejas problemáticas arqueológicas (arqueología de la producción ligada a la protoindustria y a la industrialización inglesa, arqueología del paisaje, de las transformaciones sociales, etc.).

Otro caso interesante es el holandés, presentado por J. Baart (Post-medieval archaeology in Holland), donde se ha desarrollado una intensa arqueología urbana centrada sobre el psotmedievo, especialmente en el caso de Amsterdam, donde han sido excavadas más de doscientas casas postmedievales en los últimos veinte años.

F. Verhaeghe expuso el desarrollo de la arqueología postmedieval en Bélgica (Post-Medieval archaeology in Belgium), mostrando una dinámica actividad investigadora centrada en el estudio de las ciudades, del paisaje y de la producción, mercado y consumo de cultura material, especialmente del material cerámico

Francia ha sido otro país en el que el desarrollo de la arquelogía postmedieval ha sido pionero, y que cuenta con una revista anual consolidada (RAMAGE). P. Bruneu y P.Y. Balut, en un breve texto enviado a la organización (Archéologie Postmedievale en France), ilustran las principales líneas de investigación de la disciplina, y las relaciones dialécticas con disciplinas afines.

Para el caso hispano, Fernando Amores de la Universidad de Sevilla (Arqueología postmedieval en España), ha trazado un cuadro poco alagüeño, que muestra la inmadurez, caracter ocasional y problemática de esta disciplina en nuestro país. Muchos problemas presentan paralelos estrechos con lo que sucede en Italia; otros más se ven agravados por la estructura administrativa de nuestro país, que establece distinciones profundas en la tutela y gestión del patrimonio histórico y arqueológico de comunidad a comunidad autónoma.

3. Las temáticas de la arqueología postmedieval en Italia ha constituido un repaso a las principales temáticas de estudio que van surgiendo en este país, con el fin de lograr un balance crítico de las investigaciones realizadas, proponiendo nuevas líneas de análisis. Un primer aspecto tratado, básico en un país como Italia que cuenta con una riqueza arqueológica incalculable, ha sido el de la protección del patrimonio arqueológico postmedieval a la luz de la legislación italiana ( $P$. Guzzo, La tutela del patrimonio archeologico postmedievale in Italia).

Marco Milanese, ha realizado una radiografía del estado de la arqueología postmedieval italiana (Quale archeologia postmedievale in Italia?) que ha ofrecido las claves para comprender sus deficiencias y necesidades actuales, valorando nuevas estrategias de estudio.

Entrando ya en algunas de las problemáticas más firmes dentro de esta disciplina, I. Ferrando Cabona (L'archeologia dell'edilizia: letture stratigrafiche ed indicatori cronologici) ha reflexionado sobre el desarrollo de la arqueología de la arquitectura, una de las subdisciplinas aparecidas bajo la arqueología global que ha abordado de forma pionera el estudio de los siglos posteriores al XV.

Igualmente se están produciendo importantes desarrollos en el campo de la arqueología del paisaje y de los recursos medioambientales, ilustrados por el geógrafo D. Moreno (Archeologia del paesaggio e dei sistemi agro-silvo-pastorali).

El problema del poblamiento rural en los periodos moderno y contemporáneo tuvo un incipiente desarrollo en los años 60 y 70 en Italia, si bien a partir de ese momento esta temática fue abandonada casi totalmente. J.A. Quirós Castillo (L'archeologia dell'insediamento postmedievale abbandonato) ha ofecido un panorama global de estos problemas, proponiendo nuevas líneas de estudio.

Por último, E. Giannicheda (La storia della cultura materiale)ha abordado de forma teórica los problemas derivados del estudio de la cultura material postmedieval, vastísima por cantidad y calidad respecto a otros periodos, que plantea nuevos problemas de estudio y de comprensión, al igual que interesantes relaciones con otras disciplinas que se ocupan de este sector.

4. Síntesis regionales de la arqueología postmedieval en Italia. Complementando el estudio temático de la sección anterior, se valoró la situación de la disciplina y de sus problemáticas a nivel territorial, estudiando varios casos regionales. En conjunto estaban representadas trece regiones de un total de veinte, que han mostrado nuevamente un grado de heterogeneidad, tanto en número de actuaciones, como a nivel de madurez de las distintas problemáticas propias del territorio, similar al panorama mostrado en el congreso de Albisola sobre la cerámica. Es en el norte donde la arqueología postmedieval camina en Italia de forma más decidida hacia una definición disciplinar, mientras que en el centro y sur del país la situación es esperanzadora, pero desigual.

Una mesa redonda conclusiva cerró las sesiones de trabajo. En este último debate, animado por los principales ponentes del encuentro, se han abordado los aspectos principales tratados en los cuatro dias de trabajos, definiendo algunas de las características de la disciplina en Italia en referencia al contexto europeo, y delimitando algunas líneas de investigación que deberán guiar el desarrollo de la arqueología postmedieval en los próximos años. 
Con ocasión de este encuentro ha aparecido el primer número de la revista Archeologia Postmedievale(5), que intenta proponerse como punto de referencia para todos los investigadores que trabajan en este periodo, pero tambien como instrumento de diálogo abierto a otras disciplinas sociales (antropología, historia, geografía) con las que la arqueología postmedieval se relaciona cada dia (Milanese, Quirós 1994). Uno de los aspectos que más claramente han quedado de manifiesto en el congreso de Sassari, ha sido el de la necesidad que tiene la arqueología postmedieval de enfrentarse a problemas de estudio aún arqueológicamente poco definidos (desarrollo mercantil, paso del feudalismo al capitalismo, continuidad y ruptura de las estructuras sociales en los últimos cinco siglos, etc.), que necesitan de una confrontación interdisciplinar con los estudiosos del territorio desde el siglo XVI al XX (historiadores sociales, historiadores de la cultura material y las técnicas, geógrafos históricos, antropólogos, etc.).

Al lado de este papel dinamizador, la nueva publicación intenta anular la enorme dispersión que caracteriza la producción científica italiana en ese campo. Si bien un número sustancial de las intervenciones postmedievales italianas vienen recogidas, bien como noticias, bien como ediciones preliminares o definitivas, en la revista Archeologia Medievale, son muchas las publicaciones locales o regionales donde van a parar estos estudios, ante la falta de una revista específica.
El resultado de todas estas iniciativas, destinadas a realizar una reflexión sistemática sobre una actividad arqueológica que, en cierto modo, ha crecido inconscientemente, ha llevado a la definición de unas problemáticas y unas líneas de actuación, que deberán en los próximos años guiar la actividad de los investigadores, con el fin de dotar de contenido a la arqueología postmedieval. Ha quedado claro que es lícito e imprescindible hablar de arqueología postmedieval, en tanto que no es el periodo cronológico lo que define el tipo de estudio, sino la naturaleza de la fuente arqueológica, que se desarrolla de forma diacrónica desde la prehistoria hasta el momento actual. Desde este punto de vista, puede ser tan interesante estudiar arqueológicamente un hábitat rural medieval como uno postmedieval, pues las fuentes históricas a nuestra disposición siempre son limitadas y a veces oscuras y parciales. Varias de las ponencias presentadas en estos congresos han mostrado la impensable capacidad informativa presente en el registro material, tambien para periodos muy recientes.

Como hemos resaltado anteriormente, es de gran interés subrayar los paralelos existentes entre las situaciones española e italiana en el ámbito de la arqueología postmedieval. La dependencia de la arqueología medieval y de la arqueología de intervención son quizás sólo dos de estos paralelos, pero otros más se podrán encontrar en la definición de las problemáticas y de las metodologías de intervención arqueológica para el estudio de las sociedades postmedievales.

Juan Antonio Quirós Castillo* Marco Milanese**

\section{BIBLIOGRAFÍA}

CAMEIRANA, Arrigo (1977): "La ceramica albisolese a 'taches noires', nota introduttiva". en Atti del $X$ Convegno Internationale della Ceramica, Ceramica come bene culturale, pp. 277-294.

MANNONI, Tiziano; CABONA, Danilo; FERRANDO, Isabella (1988): "Archeologia globale del territorio. Metodi e risultati di una nuova strategia della ricerca in Liguria". Castrum 2: Structures de I'habitat et occupation du sol dans les pays méditerranées. Les méthodes et l'apport de l'archéologie extensive. École Français de Rome, Casa de Velazquez, Roma-Madrid, pp. 43-58.
MILANESE, Marco; QUIROS CASTILLO, Juan Antonio (1994): "Editoriale: una proposta per l'archeologia postmedievale", Archeologia Postmedievale, I; pp. I-2.

VV.AA. (en prensa): Atti del XXV Convegno Internazionale della Ceramica, La maiolica ligure nel Cinquecento, nascita e irradiazione in Europa e nelle Americhe (1992).

5.- Para más información: Archeologia Postmedievale, Università degli Studi di Sassari, Dipartamento di Storia, Vle. Umberto 52, 07100 Sassari (Italia).

* Escuela Española de Historia y Arqueología de Roma, CSIC Via Torre Argentina 18, 00186 Roma (Italia).

** Departamento de Historia, Universidad de Sassari, Vle Umberto 52, 07100 Sassari (Italia). 